\title{
Quantitative EEG Analysis in Minimally Conscious State Patients During Postural Changes
}

\author{
A. Greco, M.C. Carboncini, A. Virgillito, A. Lanatà, Member, IEEE, \\ G. Valenza, Member, IEEE, and E.P. Scilingo, Member, IEEE
}

\begin{abstract}
Mobilization and postural changes of patients with cognitive impairment are standard clinical practices useful for both psychic and physical rehabilitation process. During this process, several physiological signals, such as Electroencephalogram (EEG), Electrocardiogram (ECG), Photopletysmography (PPG), Respiration activity (RESP), Electrodermal activity (EDA), are monitored and processed. In this paper we investigated how quantitative EEG (qEEG) changes with postural modifications in minimally conscious state patients. This study is quite novel and no similar experimental data can be found in the current literature, therefore, although results are very encouraging, a quantitative analysis of the cortical area activated in such postural changes still needs to be deeply investigated. More specifically, this paper shows EEG power spectra and brain symmetry index modifications during a verticalization procedure, from 0 to 60 degrees, of three patients in Minimally Consciousness State (MCS) with focused region of impairment. Experimental results show a significant increase of the power in $\beta$ band $(12-30 \mathbf{H z})$, commonly associated to human alertness process, thus suggesting that mobilization and postural changes can have beneficial effects in MCS patients.
\end{abstract}

\section{INTRODUCTION}

MCS is a pathological condition characterized by a severe impairment of consciousness [1]. However, MCS patients show some inconsistent but reproducible behavioral signs, which are often long enough to be clearly distinguished from simple reflexes. The MCS clinical management and treatment is still a crucial issue [2] and is usually performed using an appropriate combination of both pharmacological intervention [3], [4] and psycho-physical rehabilitation therapy [5]. Focussing on such a psycho-physical treatment, mobilization and postural changes mainly characterize the current clinical practice. Mobilization refers to the stabilization of the patient's blood circulation through physiotherapy (passive leg stepping) whereas postural changes refers to the patient's verticalization with respect to the orthostatic position. Patient's verticalization can bring several benefits such as blood circulatory dynamics, prevention of pneumonia and venous thrombosis, sympathetic activation on the autonomic nervous system. Several works in the literature suggest that passive leg movement during tilt table mobilization reduces the incidence of orthostatic dysfunction (e.g. syncope) in

A. Greco, G. Valenza, A. Lanatà and E.P. Scilingo are with the Department of Information Engineering and Research Center "E. Piaggio", Faculty of Engineering, University of Pisa, Via G. Caruso 16 - 56122, Pisa, Italy. \{a.greco, a.lanata, e.scilingo\} @centropiaggio.unipi.it; g.valenzadieee.org

M.C. Carboncini and A. Virgillito are with the Unit of Neurorehabilitation, Department of Neuroscience, University of Pisa, Italy. minimally conscious state patients [6] as well as healthy subjects [7]. Accordingly, the patients recruited in this study undergo a passive leg stepping during title table mobilization.

Mobilization is often performed while changing the patient's posture in order to avoid orthostatic dysfunction (e.g. syncope). To determine whether passive leg movement during tilt table mobilization reduces the incidence of orthostatic dysfunction in mobilization of patients being comatose or semi-comatose early after brain injury.

Despite its widespread use in other fields, quantitative studies on cortical activations have to be still investigated in patients with brain injury undergoing verticalization while leg passive stepping is carried out.

Elliott et al. demonstrated that head-up tilt improves the awareness in MCS patients [8], but only relying on behavioral changes. In this paper, we try to give an important and innovative contribution to this topic, exploring the effects of the verticalization and passive stepping in MCS patients through a quantitative analysis of the ElectroEncephaloGram (EEG), proposing an EEG power spectral analysis and symmetry index as quantitative marker of such an improvement. To this aim, previous studies on healthy subjects are taken as reference to interpret and discuss the results. In healthy subjects, in fact, postural changes influence both autonomic nervous system (e.g. [9]-[11]) and EEG activities [9]. Specifically, EEG power spectra in high frequency $(12-40 \mathrm{~Hz})$ was significantly increased in the right hemisphere [12] when passing from supine to upright. Cole [9] confirmed the hypothesis that the fall in baroreceptor firing produced by the upright posture contributes to EEG arousal in subjects who showed a normal baroreflex response to tilt. This was demonstrated by a persistent elevation of EEG beta activity.

\section{MATERIALS AND METHODS}

We studied EEG modifications in three MSC patients undergoing a verticalization and stepping protocol. Patients, aged between 56 and 73 years ( 2 females), constitute an homogeneous group in terms of focal brain injury in the left hemisphere leading to unability to walking and standing up. The diagnosis of MSC resulted from the evaluation of standardized neurobehavioral rating scales. Specifically, the Levels of Cognitive Functioning (LCF) scale [13] along with the Glasgow Outcome Scale (GOS) were used to evaluate the residual cognitive level and the motor disability level, respectively. All patients were scored as LCF and GOS equal to 3 indicating severe disability and MCS. 


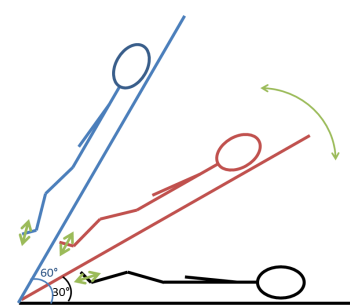

Fig. 1. Three inclinations of tilt table mobilization using the Erigo device.

The experimental protocol was performed using the Erigo device (Hocoma AG, Volketswil, Switzerland) which allows simultaneous verticalization and passive stepping. Such a device avoids orthostatic dysfunctions.

The experiment was carried out in the Brain Injury Unit, Department of Neuroscience, Cisanello Hospital, Pisa, Italy.

Patients were slowly verticalized from a supine position up to a maximum inclination of 60 degrees using the Erigo device 1. Every change of inclination lasted at least 5 minutes. The protocol timeline is defined as follows:

- 5 minutes of resting state in supine position;

- 5 minutes of supine position with passive stepping;

- 6 seconds to reach the inclination of 30 degrees;

- 5 minutes at 30 degrees of inclination with passive stepping;

- 6 seconds to reach the inclination of 60 degrees;

- 5 minutes at 60 degrees of inclination with passive stepping;

- 5 minutes of supine position with passive stepping;

During the first 5 minutes of the protocol, the clinicians performed the preliminary patient evaluations. Due to noncoherent and non-standardized timeline among patients, the related signals were excluded from further analysis. A quantitative Electroencephalogram (qEEG) analysis was carried out in terms of spectral analysis and evaluation of the Brain Symmetry Index (BSI). Recent evidences in literature, in fact, have shown the power and reliability of qEEG in the MCS clinical assessment [14].

\section{A. EEG recording}

The EEG signals were acquired using the SOMNOscreen plus system (Somnomedics, Randersacker, Germany).Signals were recorded using 13 electrodes placed on the scalp at standardized positions Fp1, Fp2, F3, F4, C3, C4, P3, P4, O1, $\mathrm{O} 2, \mathrm{Fz}, \mathrm{Cz}$ and $\mathrm{Pz}$ according to the International Standard System 10-20. The sampling frequency was set to $256 \mathrm{~Hz}$. The $\mathrm{Cz}$ electrode was used as reference whereas the two frontopolar electrodes (i.e. Fp1 and Fp2) were used to identify blinking and eye movement. An independent component analysis was performed in order to remove the blinking and other artifacts [15]. Afterwards, the signals were filtered by a $6^{\text {th }}$-order infinite impulse response band-pass filter with cut-off frequencies of $1 \div 40 \mathrm{~Hz}$. The well-known Matlab toolbox EEGLAB [16] was used for the entire processing of the EEG data.

\section{B. Spectral Analysis}

qEEG spectral analysis was performed using the Discrete Fast Fourier Transform (FFT) to estimate the power spectra within the classical frequency bandwidths: $\delta(1 \div 4 \mathrm{~Hz}), \theta$ $(4 \div 8 \mathrm{~Hz}), \alpha(8 \div 12 \mathrm{~Hz})$, and $\beta(12 \div 30 \mathrm{~Hz})$. The $\gamma$ bandwidth $(30 \div 40 \mathrm{~Hz})$ was not considered because MCS patients do not show such an electrical activity [17].

The EEG signals were divided into subsequent epochs of $8 s$ in which they can be considered stationary [18]. For each epoch, the Power Spectral Density $(P \hat{S} D)$ was estimated in each electrode and in every bandwidths. For this purpose, the periodogram method was adopted [19]. It is defined as follows:

$$
P S D(\omega)=\lim _{T \rightarrow \infty} E\left\{\frac{1}{2 T}\left|\int_{-T}^{T} x(t) e^{-j \omega t} d t\right|^{2}\right\}
$$

As the PSD reported in eq. 1 requires an infinite time of integration, we obtained the PSD periodogram estimates as follows:

$$
P \hat{S} D(\omega)=\frac{T_{s}}{N}|F F T\{x(m)\}|^{2}
$$

In order to evaluate the difference between the right and left hemisphere, the electrodes were grouped in two regions (i.e., left-region and right-region) according to the representation in fig. 2 .

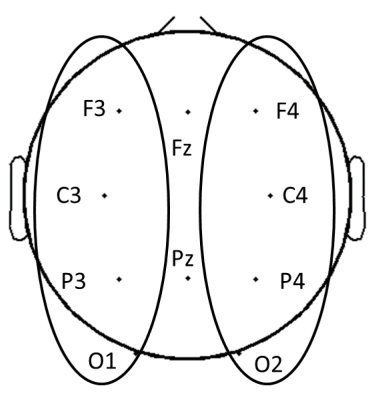

Fig. 2. The figure shows the grouping of the electrodes in the two regions (right and left)

\section{Analysis of the Brain Symmetry}

To quantify the brain symmetry changes due to verticalization, the Brain Symmetry Index (BSI) was used [20]. It is defined as the absolute value of the relative difference of the average $P \hat{S} D$ between the right and left hemisphere [20]:

$$
B S I=\left\|\frac{1}{N} \frac{1}{M} \sum_{i=1}^{N} \sum_{j=1}^{M} \frac{R_{i j}-L_{i j}}{R_{i j}+L_{i j}}\right\|
$$

where $R$ and $L$ are the $P \hat{S} D$ obtained from the $i^{t h}$ channel pair at frequency $j=1 \ldots M$ for the right and left hemisphere, respectively. The BSI is a normalized number, ranging from 0 (perfect symmetry) to 1 (maximal asymmetry). In this work, the BSI was evaluated for each frequency band. 
TABLE I

MEDIAN AND MEDIAN ABSOLUTE DEVIATION OF $P \hat{S} D$ FOR EACH BANDWIDTH AND REGION REPORTED IN LOGARITHMIC SCALE

\begin{tabular}{|c|c|c|c|c|c|c|c|c|c|}
\hline \multirow{2}{*}{ Patient } & \multirow{2}{*}{ Region } & \multicolumn{2}{|c|}{$\delta$} & \multicolumn{2}{|c|}{$\theta$} & \multicolumn{2}{|c|}{$\alpha$} & \multicolumn{2}{|c|}{$\beta$} \\
\hline & & 0 degrees & 60 degrees & 0 degrees & 60 degrees & 0 degrees & 60 degrees & 0 degrees & 60 degrees \\
\hline \multirow{2}{*}{1} & Left & $2.16 \pm 0.19$ & $2.14 \pm 0.11$ & $1.53 \pm 0.17$ & $1.64 \pm 0.11$ & $0.42 \pm 0.09$ & $1.14 \pm 0.08$ & $1.34 \pm 0.15$ & $2.19 \pm 0.13$ \\
\hline & Rigth & $2.40 \pm 0.21$ & $2.62 \pm 1.94$ & $1.77 \pm 0.11$ & $1.85 \pm 0.11$ & $0.97 \pm 0.15$ & $1.46 \pm 0.05$ & $1.91 \pm 0.12$ & $2.42 \pm 0.12$ \\
\hline \multirow{2}{*}{2} & Left & $1.91 \pm 0.1$ & $3.34 \pm 0.1$ & $1.82 \pm 0.1$ & $2.23 \pm 0.08$ & $1.28 \pm 0.27$ & $1.83 \pm 0.05$ & $0.35 \pm 0.06$ & $1.81 \pm 0.14$ \\
\hline & Rigth & $1.4 \pm 0.11$ & $2.48 \pm 0.18$ & $1.5 \pm 0.15$ & $1.69 \pm 0.1$ & $1.46 \pm 0.19$ & $2.04 \pm 0.13$ & $1.07 \pm 0.1$ & $2.01 \pm 0.06$ \\
\hline \multirow{2}{*}{3} & Left & $1.34 \pm 0.12$ & $1.72 \pm 0.1$ & $0.9 \pm 0.08$ & $1.2 \pm 0.07$ & $0.59 \pm 0.15$ & $0.71 \pm 0.07$ & $-0.19 \pm 0.15$ & $0.59 \pm 0.01$ \\
\hline & Rigth & $1.61 \pm 0.08$ & $2.16 \pm 0.17$ & $1.39 \pm .14$ & $1.7 \pm 0.07$ & $0.54 \pm 0.20$ & $0.93 \pm 0.05$ & $-0.28 \pm 0.14$ & $0.56 \pm 0.03$ \\
\hline
\end{tabular}

\section{Statistical Analysis}

Changes in $P \hat{S} D$ and BSI were evaluated using several statistical approaches considering an intra-subject analysis among the different inclinations. Accordingly, samples were obtained for each subject, each frequency band, i.e., $\delta, \theta, \alpha$, and $\beta$, and for each of the three sessions, i.e., supine position, 30 degrees, and 60 degrees. All the considered sessions included the passive stepping. Firstly, the Lilliefors test was applied in order to test the null hypothesis of having samples with gaussian distribution. In case of gaussianity, the null hypothesis of no statistical differences among sessions was tested using the Analysis of Variance (ANOVA). Otherwise, if the sample was considered to come from a not gaussian population, the Kruskal-Wallis test was applied. Statistical significance level of $p=0.05$ was considered for all tests. A post-hoc analysis was performed by means of a t-test (gaussian distributed sample) or a Mann-Whitney Utest (for non-gaussian distributed samples) considering the Bonferroni adjustment of the significance level for every pair of comparisons.

\section{EXPERIMENTAL RESULTS}

Lilliefors test showed that all data have non-gaussian distribution, therefore we used Kruskal-Wallis test for group comparison and Mann-Whitney U-test with Bonferroni adjustment for post-hoc test. We preliminarily verified whether samples of $P \hat{S} D$, evaluated for the right and left hemisphere, and BSI for each patient, for each inclination, and for each bandwidth, were belonging to the same population. Results showed that in $\delta$ and $\theta$ bandwidths no significant statistical differences were found in the $P \hat{S} D$. In $\alpha$ band there were differences among sessions in two patients out of three. BSI resulted to have no significant statistical differences in all of the three bandwidths $\delta, \theta$, and $\alpha$.

Regarding the $\beta$ band, instead, a coherent statistical difference was found in both $P \hat{S} D$, in the two hemispheres, and BSI for all of the patients. By way of illustration, in Fig. 3 the statistical comparison among the three inclinations of $P \hat{S} D$ for one patient is reported. More specifically, the posthoc analysis demonstrated that such a difference was present between the first session, in which the patients were in supine position, and the session with 60 degrees of inclination. In Tables I and II median and median absolute deviation values of $P \hat{S} D$ and BSI are reported, respectively. The choice of using median parameter is justified by non-gaussian distribution of data. Moreover, a representative topoplot showing such results is reported in Fig. 4. Concerning the difference

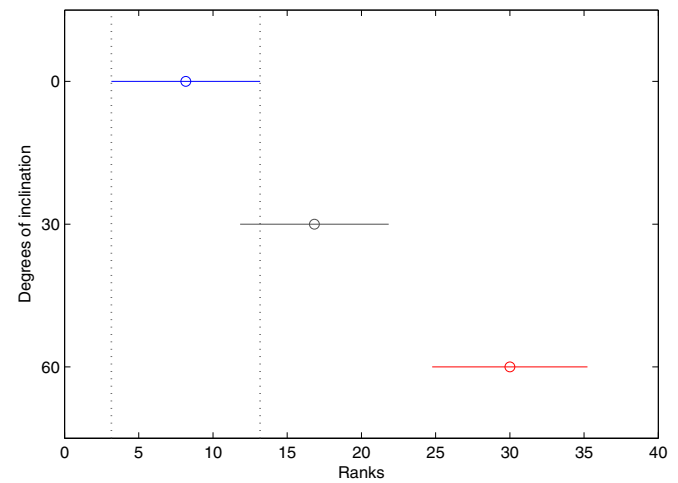

Fig. 3. Statistical comparison among the three inclinations of the tilt table for one patient.

A

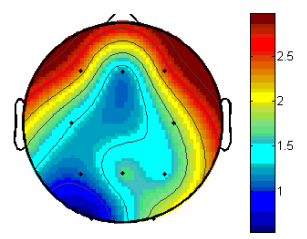

B

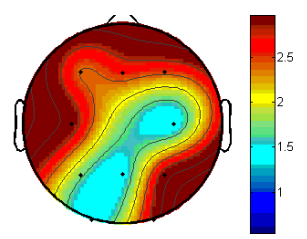

Fig. 4. Topoplot of the $P \hat{S} D$ evaluated in $\beta$ bandwidth from a representative MCS patient during the orthostatic condition (A) and after the verticalization at 60 degrees (B).

between the left and right hemisphere, the BSI results point out an increase in the left-region higher than the one in the right-region, after the transition from the supine position to the 60 degrees of inclination of the tilt table $(p<0.05)$. Moreover, looking at the $P \hat{S} D$ results in table $\mathrm{I}$, it can be noticed the largest increase in power density relative to the left hemisphere.

\section{CONCLUSions AND Discussions}

In this work, we performed a quantitative EEG analysis in patients with disorders of consciousness undergoing postural changes and passive mobilization of the lower limbs. 
TABLE II

MEDIAN AND MEDIAN ABSOLUTE DEVIATION OF BSI FOR EACH BANDWIDTH AND REGION REPORTED IN LOGARITHMIC SCALE

\begin{tabular}{|c|c|c|c|c|c|c|c|c|}
\hline \multirow{2}{*}{ Patient } & \multicolumn{2}{|c|}{$\delta$} & \multicolumn{2}{c|}{$\theta$} & \multicolumn{2}{c|}{$\alpha$} & \multicolumn{2}{c|}{$\beta$} \\
\cline { 2 - 9 } & 0 degrees & 60 degrees & 0 degrees & 60 degrees & 0 degrees & 60 degrees & 0 degrees & 60 degrees \\
\hline 1 & $0.51 \pm 0.03$ & $0.49 \pm 0.04$ & $0.48 \pm 0.02$ & $0.47 \pm 0.03$ & $0.50 \pm 0.002$ & $0.49 \pm 0.02$ & $0.46 \pm 0.007$ & $0.43 \pm 0.006$ \\
\hline 2 & $0.52 \pm 0.02$ & $0.53 \pm 0.01$ & $0.54 \pm 0.01$ & $0.55 \pm 0.01$ & $0.55 \pm 0.02$ & $0.54 \pm 0.02$ & $0.55 \pm 0.008$ & $0.46 \pm 0.01$ \\
\hline 3 & $0.47 \pm 0.02$ & $0.48 \pm 0.03$ & $0.5 \pm 0.01$ & $0.51 \pm 0.02$ & $0.39 \pm 0.01$ & $0.41 \pm 0.01$ & $0.4 \pm 0.007$ & $0.36 \pm 0.008$ \\
\hline
\end{tabular}

More specifically, the $P \hat{S} D$ estimated by means of the periodogram method, and the BSI were extracted from the EEG signals in three MCS patients. All of these patients were diagnosed with focal cortical lesion in the left hemisphere and monitored while passing from the supine to 60 degrees of inclination. Significant and coherent results were obtained considering the $P \hat{S} D$ and BSI within the $\beta$ bandwidth. Previous studies showed that EEG activities are correlated with the alertness level of healthy subjects [21], even undergoing postural changes [9]. Our results suggest that the verticalization procedure can provide beneficial effects on MCS patients showing an activation of the same cortical area. Moreover, the lateralized cerebral damage involves a decrease of the $P \hat{S} D$ in the injured region. This causes a loss of symmetry in brain activity of patients. Preliminary results of our study showed a significative increase of $P \hat{S} D$ in $\beta$ bandwidth on the injured region between 0 and 60 degrees of inclination. Specifically, at 60 degrees of inclination, the left injured region presented a higher increase than the rigth-region compensating the asymmetry in the $P \hat{S} D$. Although preliminary, these results are very encouraging and promising. The EEG activities in $\beta$, in fact, are correlated with the working memory and such increased $P \hat{S} D$ could suggest an increase in such capabilities. Future works will include a higher number of patients improving the statistical significance of results.

\section{ACKNOWLEDGMENT}

The research leading to these results has received funding from the EU FP7 n. 601165 (WEARHAP)

\section{REFERENCES}

[1] J. Giacino, S. Ashwal, Childs et al., "The minimally conscious state: definition and diagnostic criteria," Neurology, vol. 58, no. 3, 2002.

[2] C. Schnakers, A. Vanhaudenhuyse, J. Giacino, M. Ventura, M. Boly, S. Majerus, G. Moonen, and S. Laureys, "Diagnostic accuracy of the vegetative and minimally conscious state: clinical consensus versus standardized neurobehavioral assessment," BMC neurology, vol. 9, no. 1, p. 35, 2009.

[3] Y. Kim, J. Shin, and Y. An, "Effects of methylphenidate on cerebral glucose metabolism in patients with impaired consciousness after acquired brain injury," Clinical neuropharmacology, vol. 32, no. 6, p. 335, 2009.

[4] G. Valenza, M. Carboncini, A. Virgillito, I. Creatini, L. Bonfiglio, B. Rossi, A. Lanata, and E. Scilingo, "Eeg complexity drug-induced changes in disorders of consciousness: A preliminary report," in Engineering in Medicine and Biology Society, EMBC, 2011 Annual International Conference of the IEEE. IEEE, 2011, pp. 3724-3727.

[5] L. Elliott and L. Walker, "Rehabilitation interventions for vegetative and minimally conscious patients," Neuropsychological rehabilitation, vol. 15 , no. 3-4, pp. 480-493, 2005.
[6] M. Luther, C. Krewer, F. Muller, and E. Koenig, "Comparison of orthostatic reactions of patients still unconscious within the first three months of brain injury on a tilt table with and without integrated stepping. a prospective, randomized crossover pilot trial," Clinical rehabilitation, vol. 22, no. 12, pp. 1034-1041, 2008.

[7] L. Chi, K. Masani, M. Miyatani, T. Adam Thrasher, K. Wayne Johnston, A. Mardimae, C. Kessler, J. Fisher, and M. Popovic, "Cardiovascular response to functional electrical stimulation and dynamic tilt table therapy to improve orthostatic tolerance," Journal of Electromyography and Kinesiology, vol. 18, no. 6, pp. 900-907, 2008.

[8] L. Elliott, M. Coleman, A. Shiel, B. Wilson, D. Badwan, D. Menon, and J. Pickard, "Effect of posture on levels of arousal and awareness in vegetative and minimally conscious state patients: a preliminary investigation," Journal of Neurology, Neurosurgery \& Psychiatry, vol. 76, no. 2, pp. 298-299, 2005.

[9] R. Cole, "Postural baroreflex stimuli may affect eeg arousal and sleep in humans," Journal of Applied Physiology, vol. 67, no. 6, pp. 2369$2375,1989$.

[10] G. Valenza, L. Citi, E. Scilingo, and R. Barbieri, "Using laguerre expansion within point-process models of heartbeat dynamics: A comparative study," in Engineering in Medicine and Biology Society (EMBC), 2012 Annual International Conference of the IEEE. IEEE, 2012, pp. 29-32.

[11] — , "Instantaneous bispectral characterization of the autonomic nervous system through a point-process nonlinear model," in World Congress on Medical Physics and Biomedical Engineering, IFMBE Proceedings, vol. 39, 2012, pp. 530-533.

[12] L. Chang, J. Lin, C. Lin, K. Wu, Y. Wang, and C. Kuo, "Effect of body position on bilateral eeg alterations and their relationship with autonomic nervous modulation in normal subjects," Neuroscience Letters, vol. 490, no. 2, pp. 96-100, 2011.

[13] B. Jennett, J. Snoek, M. Bond, and N. Brooks, "Disability after severe head injury: observations on the use of the glasgow outcome scale." Journal of Neurology, Neurosurgery \& Psychiatry, vol. 44, no. 4, pp. 285-293, 1981.

[14] E. Kobylarz and N. Schiff, "Neurophysiological correlates of persistent vegetative and minimally conscious states," Neuropsychological rehabilitation, vol. 15, no. 3-4, pp. 323-332, 2005.

[15] T. Jung, S. Makeig, C. Humphries, T. Lee, M. Mckeown, V. Iragui, and T. Sejnowski, "Removing electroencephalographic artifacts by blind source separation," Psychophysiology, vol. 37, no. 2, pp. 163-178, 2000.

[16] A. Delorme and S. Makeig, "Eeglab: an open source toolbox for analysis of single-trial eeg dynamics including independent component analysis," Journal of neuroscience methods, vol. 134, no. 1, pp. 9-21, 2004.

[17] P. Hansotia, "Persistent vegetative state: review and report of electrodiagnostic studies in eight cases," Archives of neurology, vol. 42, no. 11, p. 1048, 1985.

[18] C. Ehlers, J. Havstad, D. Prichard, and J. Theiler, "Low doses of ethanol reduce evidence for nonlinear structure in brain activity," The Journal of neuroscience, vol. 18, no. 18, pp. 7474-7486, 1998.

[19] J. Bronzino et al., The biomedical engineering handbook. CRC press Boca Raton, FL, 1995, vol. 1.

[20] M. van Putten and D. Tavy, "Continuous quantitative eeg monitoring in hemispheric stroke patients using the brain symmetry index," Stroke, vol. 35, no. 11, pp. 2489-2492, 2004.

[21] M. Dijkman, N. Sachs, E. Levine, M. Mallis, M. Carlin, K. Gillen, J. Powell, S. Samuel, J. Mullington, M. Rosekind et al., "Effects of reduced stimulation on neurobehavioral alertness depend on circadian phase during human sleep deprivation," Sleep Res, vol. 26, p. 265, 1997. 